Stephen D. Whitlow, Michael C. Dorneich, Harry B. Funk, Christopher A. Miller (2002). "Providing Appropriate Situation Awareness within a Mixed-Initiative Control System," to appear in The Proceedings of the 2002 IEEE International Conference on Systems, Man, and Cybernetics, Hammamet, Tunesia, 6-10 October 2002.

\title{
Providing Appropriate Situation Awareness within a Mixed-Initiative Control System
}

\author{
Stephen D. Whitlow ${ }^{1}$, Michael C. Dorneich \\ Honeywell Laboratories -3660 Technology Drive, Minneapolis, MN 55418 \\ Harry B. Funk, Christopher A. Miller \\ Smart Information Flow Technologies (SIFT)- 2119 Oliver Avenue South \\ Minneapolis, MN 55405 \\ Submitted to: \\ IEEE International Conference on Systems, Man and Cybernetics 2002, \\ [T6] Human-Machine Systems Track, \\ October 6-9, 2002 in Hammamet, Tunisia
}

\begin{abstract}
The future of air combat relies on humans controlling large teams of unmanned combat air vehicles (UCAVs) within a dynamic battle environment. Under the DARPA Mixed Initiative Control of Automata (MICA) program, we have been challenged to design a system that empowers a human operator to control teams of up to thirty UCAVs. To address these challenges we are designing an interaction system that defines and provides adequate situation and automation awareness without overloading human operators to the point where their performance degrades gracelessly.

The proposed mixed initiative system is situated within a complex and highly dynamic information space that could easily overload the multi-tasking human operators. Dozens of system parameters could be updated thousands of times during a typical mission so it is neither feasible nor prudent for human operators to maintain complete situation and automation awareness. This interaction system will provide appropriately abstracted situation awareness and notification capability that includes: general mission monitoring and automation awareness; task specific information requirements; and user initiated information requests. Our approach involves defining adequate situation awareness as a function of mission phase, human operator role, and abstracted information required to oversee tasked UCAV.
\end{abstract}

Keywords: mixed initiative, situation awareness, command and control, supervisory control

\section{INTRODUCTION}

Throughout the history of human-automation systems, designers have struggled to define and provide appropriate levels of situation and

automation awareness. The pitfalls of inadequate human awareness within complex human- automation systems have been well documented including: automation surprises; mode confusion; and overreliance on automation (Riley, 1996; Parasuraman \& Riley, 1997).

However, as human-automation control systems increase in complexity and reduced manning initiatives proceed, future human operators will be severely challenged in maintaining awareness in dynamic, multi-tasking control environments. This challenge is further complicated by the propensity of automation designers to automate as much as is technically possible then relegate the human to the role as a passive monitor for which they are woefully ill suited. Some common problems with passive monitoring of automation include over-reliance on automation decisions (Parasuraman \& Riley, 1997) and susceptibility to brittle models (Smith et al., 1997). We have been particularly cognizant of the potential for awareness issues within this environment since it could easily satisfy Sarter and Woods' criteria for automation problems: automation with high levels of authority and autonomy; poor mental models of automation; and low observability if automation (1997).

Previous studies have demonstrated that a majority of workload within a multi-tasking environment is dedicated to "creating, maintaining, and updating" situation awareness (MacMillan et al., 1997). Mixed-initiative control of tasked subordinates will be a fastpaced, multi-tasking work environment

\footnotetext{
${ }^{1}$ Contact Author: Stephen Whitlow, Honeywell Laboratories, 3660 Technology Drive, Minneapolis, MN 55418, phone: (612) 951-7430, fax: (612) 951-7438, e-mail: stephen.whitlow@ honeywell.com
} 
Stephen D. Whitlow , Michael C. Dorneich, Harry B. Funk, Christopher A. Miller (2002). "Providing Appropriate Situation Awareness within a Mixed-Initiative Control System," to appear in The Proceedings of the 2002 IEEE International Conference on Systems, Man, and Cybernetics, Hammamet, Tunesia, 6-10 October 2002.

characterized by rapid task switching that will severely challenge the cognitive capabilities of human operators.

One of the benefits of human-automation systems is that automation can assume responsibility for tasks where human input either does not add value or there is not sufficient time or workload capacity to elicit a human response; this frees human workload up so they can focus on value-added activities such as:

- Exercising their legal authority and fiduciary responsibility

- Creatively and flexibly solving problems

- Expressing their unique knowledge

- Visual information processing

- Abstraction and projection

- Analogical reasoning

- Responding to unforeseen circumstances

- Mitigating inevitable system brittleness

- Exercising system oversight including judgements about how trustworthy and effective is the automation

For example, in this domain it would not make any sense for human supervisors to continuously monitor current altitude and heading, much less exert joystick control, for more than 1 or 2 vehicles at a time; instead of expending critical workload on tasks that are below their value region, they could focus on value-added tasks such as mission goal awareness, risk monitoring, problem solving, detecting enemy deception, leveraging un-represented data sources, and determining enemy disposition.

\section{APPROACH}

Our challenge is to design a complex mixedinitiative control system that enables human supervisors to do the following efficiently and effectively:

- Generate mission packages

- Task autonomous subordinates

- Maintain oversight

- Respond decisively in a dynamic battle environment
We concluded that absolute awareness would be prohibitively costly in terms of human workload; instead we will deliver adequate and appropriate awareness to support the effective oversight of tasked autonomous subordinates, such as UCAVs. Critical components of this approach include:

- Maintaining an active and engaged human supervisor

- Delegation of some tasks to automation

- Automation observability and projection

- Information abstracted and distilled to the appropriate level for oversight

In order to maintain an active and engaged human supervisor, system designers must initially define their role based on their responsibilities and capabilities and not as an after-thought to automation development. Their appropriately defined role can be characterized as follows:

- Involved and integral in the system workflow

- Aware of the environment, automation actions and intentions and rationale

- Active involvement in system workflow not only support human awareness it also fosters the development of mental models of automation functionality

Although this approach will likely produce occasional, local, sub-optimal system performance, it is nonetheless an important safeguard for long-term overall system performance

Since we cannot say a priori the type and extent of automation that would be optimal, the delegation of tasks should be conducted within an adjustable autonomy framework. Our innovative approach calls for a moving middle ground of human-automation interaction: adjustable autonomy that can assume more or fewer of the duties depending on the situation. The proposed system will support human involvement in the process at whatever level of detail is necessary for optimal achievement of 
Stephen D. Whitlow , Michael C. Dorneich, Harry B. Funk, Christopher A. Miller (2002). "Providing Appropriate Situation Awareness within a Mixed-Initiative Control System," to appear in The Proceedings of the 2002 IEEE International Conference on Systems, Man, and Cybernetics, Hammamet, Tunesia, 6-10 October 2002.

mission objectives. This contrasts with most work in autonomous systems, in which the level of interactions between human and automation are fixed by design. This framework would allow an overloaded human supervisor to delegate some tasks to automation. Likewise, if a change in world state requires a response faster than estimated human decision making latency, automation will respond then inform the human supervisor afterwards.

In order to maintain adequate automation awareness, human supervisors should be provided accessible feedback on automation status, behavior, intentions, and limitations; however, it's not just about the availability and quality of feedback, it is also about how much cognitive effort is required to maintain "actionable" awareness that is integrated with knowledge about current and future options. Automation awareness can be improved by:

- Providing appropriately abstracted status information to minimize effort

- Include meaningful confirmation that user input was understood

- Provide salient mode transitions

- Preview of future automation activities (Sarter \& Woods, 1997).

When humans task semi-autonomous subordinates, such as UCAVs, the information requirements for effective oversight is a subset or abstracted version of the totality of information generated during the mission execution of a UCAV. Our approach to maintaining adequate situation and automation awareness was first to identify those classes of information that support effective oversight, such as:

1. General mission monitoring and automation awareness: status of the of mission goals, highly observable automation state information, and factors that could cause plan failure (Cohen et al., 1998)

2. Mission phase or task specific information requirements: includes CCIRs as a function of mission phase, specific information required to make scheduled or anticipated decisions, and factors that could cause sub-plan failures

3. User initiated information requests--does not constrain user review of information by a priori assumptions about what is relevant

4. We could leverage the Playbook task models by linking them with information requirements. Information is needed when it enables some goal or supports task performance. Accordingly, if the human supervisor delegates some task to automation they are, in effect, informing the system that they do not need execution information requirements for those tasks, but they do want success and progress information.

Adequate situation and automation awareness is the union of current and near-term task information requirements as well as a general set of mission performance information that correspond to Vincente and Rasmussen's functional purpose layer (1992).

\section{INTERACTION CONCEPTS}

We have also proposed several interaction concepts that will support adequate awareness, including:

- User Initiated Notification:

- CCIR schedule by mission phase

- Playbook interaction system

- Brittle Model Mitigation

User Initiated Notification. Given the challenging multi-tasking environment, we should provide human supervisor the means to delegate some of those monitoring tasks where there is no value-added in requiring the human to actually conduct the task. Accordingly, User Initiated Notification (UIN) allows operators to define a priori those condition under which they would like to be notified and how the system should notify them (Guerlain \& Bullemer, 1996). UIN software allows operators to define personal, context-sensitive monitors of computer-based information, thereby improving their situation awareness and reducing workload. A monitor may either notify users when a specified condition has occurred or it 
Stephen D. Whitlow , Michael C. Dorneich, Harry B. Funk, Christopher A. Miller (2002). "Providing Appropriate Situation Awareness within a Mixed-Initiative Control System," to appear in The Proceedings of the 2002 IEEE International Conference on Systems, Man, and Cybernetics, Hammamet, Tunesia, 6-10 October 2002.

may initiate other processes, depending on the users' goal. For example, the human supervisor could define a UIN to notify her when any of her tasked assets is low on fuel and has not yet achieved its objective. We will support a library of alerts that could be inherited by any mission or to which you could add new alerts specific to a given mission.

CCIR schedule by mission phase. Given the nature of a given mission, we can a priori predict some of the information that is critical for effective oversight. This is referred to as Commander's Critical Information Requirements (CCIRs). We will present a schedule of CCIRs by mission phase to be reviewed and edited by human supervisors prior to mission execution. Human supervisors can further streamline their work process by eliminating those default information requirements that are not relevant to the current mission variant; likewise the process of reviewing the schedule encourages them to cognitively simulate the mission which will increase the probability that they will think of additional information requirements. This cognitive pre-processing also primes them for decision points during mission execution.

Playbook interaction system. We will leverage the common training and knowledge of warfighters via our Playbook interaction system. The human will communicate instructions as goals, tasks, partial plans, or constraints via Playbook expressed in a Common Task Language (CTL). These are the same semantics and methods used to express commander's intent in training approaches for U.S. battalion commanders (Klein, 1998; Shattuck, 1995). The great strength of this approach is that it is familiar to humans and interpretable by a modelbased control system; therefore, there is no need for translations when communicating control information from automaton to human, or for providing guidance from human to automaton. It is the ability of the human to express intent at a context-appropriate level of abstraction, with the inherent CTL support for situation and automation awareness, that will enable the adjustable autonomy system to ensure that mission objectives are met in dynamic, uncertain, and adversarial domains.
Playbook will provide a familiar cognitive structure for representing the mission situation that is based on military tasks and domain models (Miller \& Goldman, 1997). Playbook will support both mission planning and monitoring in a familiar framework that will reduce the workload required to maintain awareness during mission execution.

Brittle Model Mitigation. Smith et al. postulated that automated systems will always be susceptible to "brittle model" as long as the world is not a closed system and resources are limited (1997). We have proposed a framework to minimize this negative, and unfortunately inevitable, impact of brittle model within automated systems. Oftentimes systems are limited by the availability of data for known classes of relevant information-- designers know about its value but do not have the means to automatically collect, represent, or reason about it.

Our approach it to first identify and represent within domain models those information classes that are known to be relevant to system decisions--even if we know that we will never be able to populate those classes with data. Therefore, the automated reasoner is "aware" of relevant factors within the domain model even though it could not reason about it in the absence of data. Accordingly, when presenting any automated decision, the system could also present the user those relevant classes of information that it did not consider. Having this information will improve the human supervisor's ability to evaluate automated decisions and identify the limitations inherent to brittle models; moreover, by specifically identifying such information, human supervisors can improve automated solutions with what they know about the un-included information classes.

Insertion of future decision points. We will empower human supervisor to define decision points within the mission execution schedule during mission planning. This will allow users to schedule anticipated interactions and predefine any relevant information requirements. 
Stephen D. Whitlow , Michael C. Dorneich, Harry B. Funk, Christopher A. Miller (2002). "Providing Appropriate Situation Awareness within a Mixed-Initiative Control System," to appear in The Proceedings of the 2002 IEEE International Conference on Systems, Man, and Cybernetics, Hammamet, Tunesia, 6-10 October 2002.

By defining these scheduled human participation points, users are effectively "primed" for those decisions (Klein, 1993)

\section{DISCUSSION}

This paper represents our vision for efficient and engaged human supervision within a complex mixed-initiative system. Many of these concepts are under parallel development on a variety of programs. It is our contention that many of these elements are modular and individually can add value to any system. That is to say it is not required to implement the entire vision to see benefit in terms of improving awareness while managing workload.

\section{ACKNOWLEDGEMENTS}

The authors would like to thank Jim Richardson for his support as Honeywell Labs' program manager on the DARPA MICA program.

\section{REFERENCES}

Cohen, M. S., Parasuraman, R., and Freeman, J.T. (1998). Trust in Decision Aids: What is it and how can it be improved? Proceedings of the 1998 Command and Control Research \& Technology Symposium, Monterey, CA.

Guerlain, S. \& Bullemer, P. (1996). User-initiated notification - a concept for aiding the monitoring activities of process control operators. Proceedings of the Human Factors and Ergonomics Society 40th Annual Meeting, Sept. 2-6, 1996. Santa Monica, CA. p.283-287.

Klein, G. A. (1993). A recognition-primed decision (RPD) model of rapid decision making. In Klein, G. A., Orasanu, J., Calderwood, R. \& Zsambok, C.E. (Eds.), Decision making in action: Models and methods. Norwood, NJ: Ablex Publishing Corporation. 138-147

Klein, G. (1998). Sources of Power: How People Make Decisions. MIT Press; Cambridge, MA

MacMillan, Deutch, and Young (1997). A comparison of alternatives for automated decision support in a MT environment. Human Factors and Ergonomic Society Proc. $41^{\text {st }}$ Meeting .

Miller, C., \& Goldman, R. (1997). “"Tasking' Interfaces: Associates that know who's the boss".
Proceedings of the 4th USAF/RAF/GAF Conference on Human/Electronic Crewmembers. Kreuth, Germany.

Parasuraman, R., and Riley, V. (1997). "Humans and Automation: Use, Misuse, Disuse, Abuse." Human Factors, 39(2), 230-253.

Riley, V. (1996). "Operator reliance on automation: Theory and data." In R. Parasuraman and M. Mouloua (Eds.), Automation and Human Performance: Current Theory and Applications. Lawrence Erlbaum: Hillsdale, NJ, 1996, 19-36.

Sarter N. \& Woods, D.D. (1997). Team Play with a Powerful and Independent Agent: Operational Experience and Automation Surprise on the Airbus A320. Human Factors, 1997, 39(4), 553-569.

Shattuck, L. (1995). Communication of Intent in Distributed Supervisory Control Systems. Unpublished dissertation. The Ohio State University, Columbus, $\mathrm{OH}$..

Smith, Phillip J.; McCoy, Elaine C.; Layton, Charles. "Brittleness in the design of cooperative problemsolving systems: the effects of user performance". IEEE Transaction on Systems, Man, and Cybernetics--Part A: Systems and Humans, Vol. 27, NO. 3, May 1997

Vincente, K. \& Rasmussen, J. (1992). Ecological interface design: Theoretical foundations. IEEE Transactions on Systems, Man and Cybernetics. SMC-22, 589-606. 